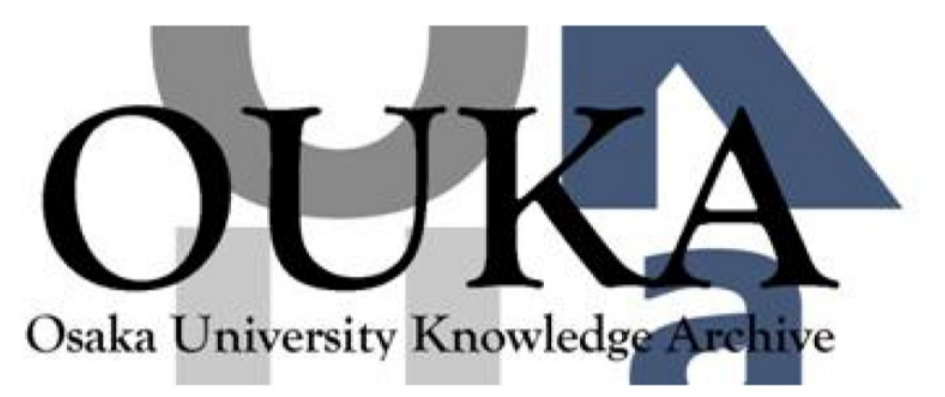

\begin{tabular}{|c|l|}
\hline Title & $\begin{array}{l}\text { Electro-optic switching in spin-coated } \\
\text { ferroelectric mesomorphic polymer films and its } \\
\text { analysis }\end{array}$ \\
\hline Author(s) & $\begin{array}{l}\text { Uto, Sadahito; Moritake, Hiroshi; Ozaki, } \\
\text { Masanori et al. }\end{array}$ \\
\hline Citation & Journal of Applied Physics. 79(8) p. 4444-p. 4449 \\
\hline Issue Date & $1996-04-15$ \\
\hline oaire:version & VoR \\
\hline URL & https://hdl. handle.net/11094/75856 \\
\hline rights & \\
\hline Note & \\
\hline
\end{tabular}

Osaka University Knowledge Archive : OUKA

https://ir. Library. osaka-u. ac. jp/

0saka University 


\section{Electro-optic switching in spin-coated ferroelectric mesomorphic polymer films and its analysis}

Cite as: Journal of Applied Physics 79, 4444 (1996); https://doi.org/10.1063/1.361754

Submitted: 19 October 1995 . Accepted: 19 January 1996 . Published Online: 17 August 1998

Sadahito Uto, Hiroshi Moritake, Masanori Ozaki, Katsumi Yoshino, Kent Skarp, and Bertil Helgee

View Online

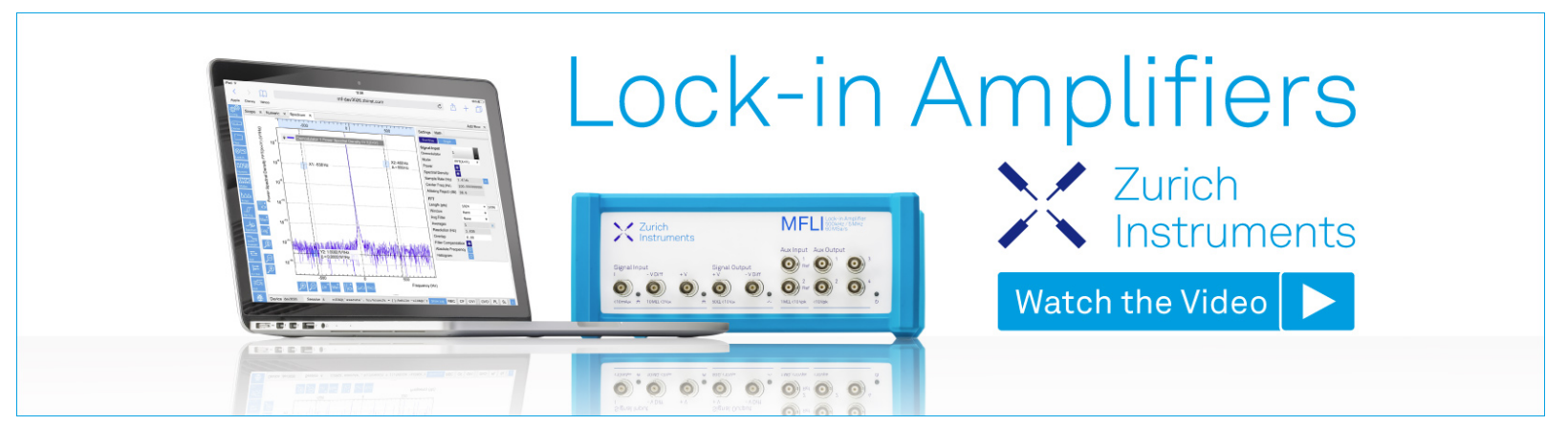




\title{
Electro-optic switching in spin-coated ferroelectric mesomorphic polymer films and its analysis
}

\author{
Sadahito Uto, Hiroshi Moritake, Masanori Ozaki, and Katsumi Yoshino \\ Department of Electronic Engineering, Faculty of Engineering, Osaka University, 2-1 Yamada-Oka, Suita, \\ Osaka 565, Japan \\ Kent Skarp \\ Department of Physics, Chalmers University of Technology, S-412 96 Goteborg, Sweden \\ Bertil Helgee \\ Department of Polymer Technology, Chalmers University of Technology, S-412 96 Goteborg, Sweden
}

(Received 19 October 1995; accepted for publication 19 January 1996)

\begin{abstract}
Thin films of a chiral side-chain polymer with a ferroelectric phase were deposited on a substrate by spin coating. Electro-optic switching in the films were observed by applying an external field using in-plane electrodes. Characteristics of the switching are presented, together with an analysis of the switching dynamics that takes into account the inhomogenous field distribution in the active area. (C) 1996 American Institute of Physics. [S0021-8979(96)10208-3]
\end{abstract}

\section{INTRODUCTION}

Thin organic films that are functionalized for specific applications are of growing interest for microelectronic and optoelectronic applications. Of particular interests are molecularly ordered films with polar order. Examples of such films are Langmuir-Blodgett layers, ${ }^{1}$ electrically poled polymer films, and self-assembled mono- and multilayers. ${ }^{2}$ A different route is to take advantage of the molecular order inherent in the liquid crystalline (or mesomorphic) state. In this way one can obtain thermodynamically stable polar orientation of mesogenic groups. By using a ferroelectric liquid crystalline polymer we were recently able to demonstrate how to make extremely well-ordered thin films with interesting electro-optic characteristics. ${ }^{3,4}$ In these two articles we reported preparation methods and basic electro-optic effects in free-surface spin-coated thin films of ferroelectric liquid crystalline polymers. We could thus establish the basic concept of spin-coated macroscopic films consisting of several hundred to thousands of smectic layers with perfect layer stacking, and also demonstrate several important macroscopic physical properties of such mesomorphic polymer films.

In this article we analyze the dynamics of the director cone reorientation in the geometry, in which smectic layers are parallel to the substrate, adopted by the polymer after spin coating. After presenting a switching model for the case of homogenous electric field, we discuss how to account for the inhomogenous field distribution in the active area. The result from these considerations is that there will be a distribution of director orientations over the electro-optic measurement spot, which can be incorporated in the switching model by one spatial integration at every time instant of the switching. The model is compared to experimental results, and we also present switching properties in a multielectroded film.

\section{FILM PREPARATION}

The polymer ferroelectric liquid crystal (PFLC) used in this work was synthesized as described in Ref. 5, where basic ferroelectric, electroclinic, and pyroelectric properties of the polymer are also given. The molecular structure of the PFLC is shown in Fig. 1, together with phase transition temperatures.

Free-surface films of the PFLC were prepared as follows. A conductive In-Sn (ITO)-coated glass substrate (10 $\mathrm{mm} \times 10 \mathrm{~mm}$ ) whose surface was treated with a silane coupler (AY43-021, Toray Dow Corning Silicone) was set on a turntable of a spin-coating machine. The solution of PFLC (740 mg dissolved in $2.5 \mathrm{~cm}^{3}$ of chloroform was dropped over the substrate. Excess solution was removed by revolving the substrate at $250 \mathrm{rpm}$ for $1 \mathrm{~s}$, and then $1500 \mathrm{rpm}$ for 15 s. A PFLC film was obtained on the substrate. The typical thickness of the films estimated by interference fringe measurement was about $5 \mu \mathrm{m}$. The thickness of the film depends on the concentration of PFLC in the solution. For example, films with thickness of about $1 \mu \mathrm{m}$ were prepared using a solution of $740 \mathrm{mg}$ of PFLC dissolved in $10 \mathrm{~cm}^{3}$ of chloroform.

By observing the spin-coated films by an optical microscope in the chiral smectic $C$ phase ( $\mathrm{Sm} C^{*}$ phase), an alignment in which the smectic layers were parallel to the glass substrate was confirmed. An electrode pattern consisting of two parallel conductive areas insulated by a stripe of $0.5 \mathrm{~mm}$ width etched onto the ITO substrate beforehand [see Fig. 2(a)]. An electric field was applied parallel to the smectic layers with the etched electrode pattern.

The polymer film was placed between crossed polarizers, and the time dependence of light transmission was recorded when a square-wave voltage was applied to the cell. Results from these measurements are discussed in Sec. III. In addition to the single-gap substrate, we also studied films made on multielectroded ITO substrates, cf. Fig. 2(b).

\section{EXPERIMENTAL RESULTS AND DYNAMICAL MODEL}

The electro-optic effects in the spin-coated films in the chiral smectic $C$ phase are of several different kinds: transient scattering mode and unwinding of helix or uniform 


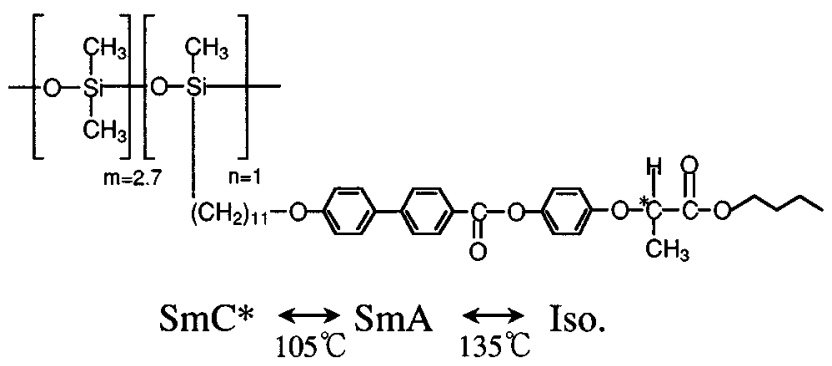

FIG. 1. Molecular structure and phase transition temperatures of the ferroelectric polymer liquid crystal used in this study.

cone reorientation. ${ }^{3}$ In this article, we discuss the conereorientation mode in some detail. In Ref. 6, a conventional sandwich cell filled with low-molar mass smectic $C^{*}$ liquid crystal was used to record the switching characteristics in the cell in which smectic layers are parallel to the substrate.

Experimental transmission curves of the spin-coated film between crossed polarizers, with an angle $\pi / 4$ between the polarizer and an external field are shown in Fig. 3 for a 0.5 mm electrode gap. The temperature was $80^{\circ} \mathrm{C}$, and applied voltage 60 V. In Fig. 3(a) film thickness was $1 \mu \mathrm{m}$, and in Fig. 3(b) $5.3 \mu \mathrm{m}$. Probably sample was a little tilted to give different states before and after switching.

The analysis of the electro-optic response in the polymer film originating from cone reorientation of the director after field reversal can be done using a phenomenological model based on a driving ferroelectric torque and a counteracting

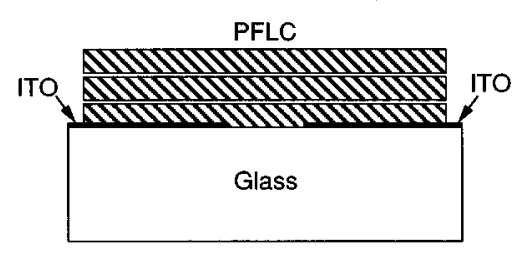

(a)

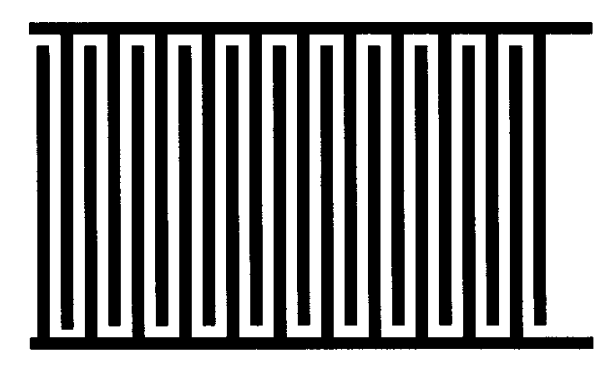

(b)

FIG. 2. (a) Arrangement of smectic layers in the orientation used in this study on the $0.5 \mathrm{~mm}$ electrode gap substrate. (b) The electrode pattern in the multielectroded ITO substrate.
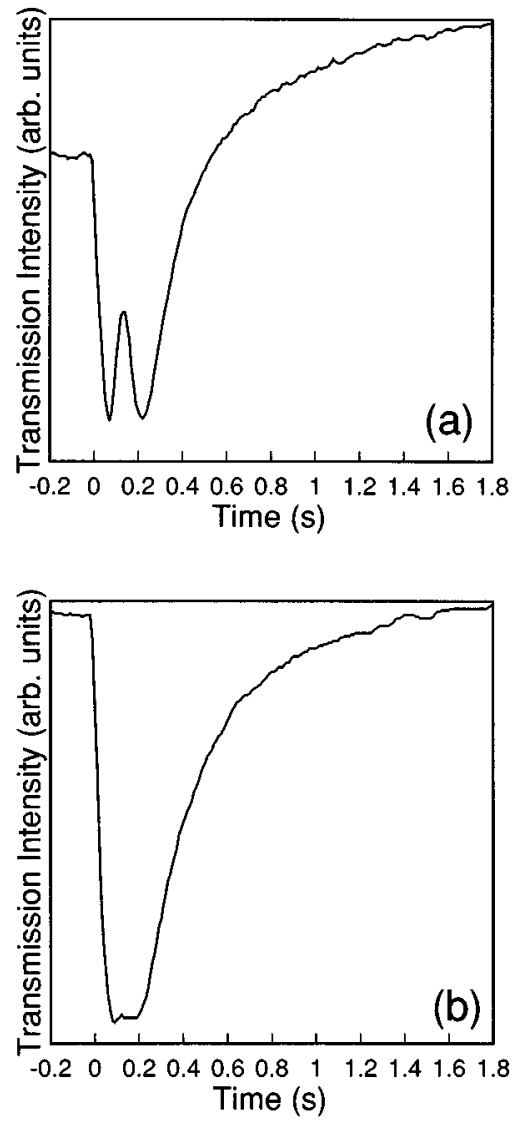

FIG. 3. Experimental recordings of optical transmission between crossed polarizers with $\alpha=\pi / 4$ : (a) Results for a film of thickness $1 \mu \mathrm{m}$; (b) results for a film of thickness $5.3 \mu \mathrm{m}$.

viscous torque acting on the director. Since applied fields are low, ${ }^{3}$ and much lower than in conventional sandwich-type cells in which smectic layers of ferroelectric liquid crystal are perpendicular to the substrates, ${ }^{7}$ we do not include the dielectric torque on the director, which is important only at high fields because it is proportional to $E^{2}$. For a complete analysis of the response of the film to an applied field, several factors should be taken into account, such as the existence of two different surfaces: air-polymer and polymerglass, and the field inhomogeneity in the active area. Surface effects lead to a director profile perpendicular to the film. We choose to neglect this effect in the present analysis, because at the free surface, the aligning force is very weak, and the orientation at the glass interface also makes the influence of that boundary weak. The existence of two extremely thin boundary layers with slightly different director orientations will change the observed transmitted intensity negligibly, compared to the uniform reorientation in the bulk of the film. Consequently, we confine our analysis to the influence of field inhomogeneity along the film. Before considering this effect, we present the case of uniform field, which clarifies the basic underlying phenomena.

\section{A. Uniform electric field}

For a uniform field, we consider the field-induced reorientation of the director to also be uniform, and we model the 


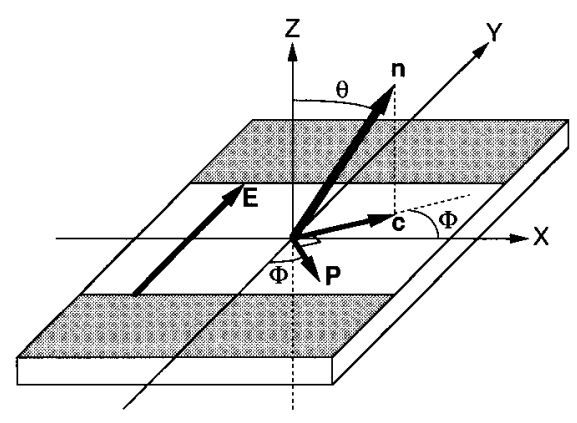

FIG. 4. Definition of coordinate system and geometry of the cone switching in the smectic $C^{*}$ phase.

dynamics of the reorientation which occurs upon field reversal. Referring to Fig. 4, which shows the orientation of the director at time $t$ after field reversal, the director $\mathbf{n}$ makes an angle $\Phi$ with the $x$ axis, and the polarization $\mathbf{P}$ makes the same angle with the $y$ axis. The projection of $\mathbf{n}$ on the $x y$ plane is the smectic $C$ director c. The smectic tilt angle is denoted $\theta$, and the electric field is shown along the positive $y$ axis. The $\mathbf{c}$ and $\mathbf{P}$ vectors will rotate counterclockwise until $\mathbf{P}$ becomes parallel to $\mathbf{E}$. This rotation is driven by the ferroelectric torque

$$
\Gamma^{F}=P \times E \times \sin \Phi,
$$

which is zero for $\Phi=0$ and $\pi$, and maximal for $\Phi=\pi / 2$. In order for the switching to start, it is necessary to assume an initial small angle $\Phi_{0}$ between the director and the $x$ axis. The reorientation of the director is counteracted by the viscous torque

$$
\Gamma^{V}=-\gamma_{\Phi} \times \frac{\partial \Phi}{\partial t},
$$

where $\gamma_{\Phi}$ is the rotational viscosity coefficient for the $\Phi$ rotation. As discussed above, we neglect other influences on the director, and the torque balance equation gives

$$
\Gamma^{F}+\Gamma^{V}=0,
$$

which yields the equation of motion for the $\mathbf{c}$ director

$$
P \times E \times \sin \Phi=\gamma_{\Phi} \times \frac{\partial \Phi}{\partial t} .
$$

This equation is integrated to give

$$
t=\frac{\gamma_{\Phi}}{P \times E} \times \ln \left[\frac{\tan (\Phi / 2)}{\tan \left(\Phi_{0} / 2\right)}\right],
$$

which is the equation relating $\Phi$ and $t$, with the initial angle $\Phi_{0}$ as parameter. It is more convenient for the further discussion to use $\Phi$ as an explicit function of $t$ :

$$
\Phi(t)=2 \quad \arctan \left[e^{t / \tau} \times \tan \left(\Phi_{0} / 2\right)\right],
$$

where $\tau=\gamma_{\Phi} / P E$ is the response time for the cone motion of the director upon field reversal. ${ }^{8}$ The transmitted light intensity between crossed polarizers, with the angle $\alpha$ between polarizer and $x$ axis, is given by

$$
T(t)=\sin ^{2}[2 \Phi(t)+2 \alpha] \times \sin ^{2}(\delta / 2),
$$

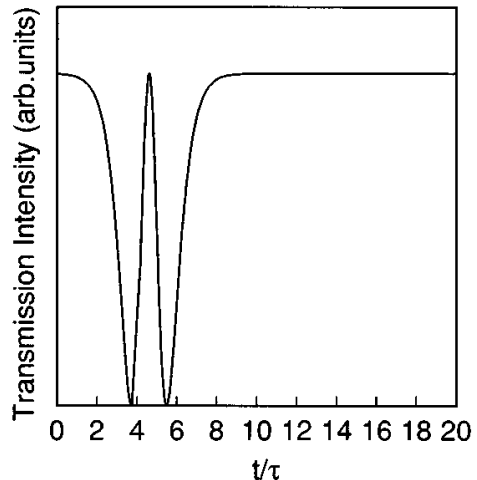

FIG. 5. Results from a plotting of the transmission vs time using Eq. (9) with $\alpha=\pi / 4$.

where $\delta=2 \pi d \Delta n / \lambda$ is the phase factor. It has maximum when $\delta=\pi$ (half-wave plate). Normally the thickness of the polymer films is too small to match this condition. For a birefringence of 0.2 it would take a film of thickness $d$ $=4 \mu \mathrm{m}$ to fulfill the half-wave plate condition, assuming a tilt angle of $37 \mathrm{deg}$ as for the polymer used in this work. Although we have made $5 \mu \mathrm{m}$ films or even thicker, normally films of 1-2 $\mu \mathrm{m}$ thickness are used in the electro-optic studies. The transmitted intensity in these films is sufficient for the electro-optic measurements. For the present investigation of switching in the smectic $C^{*}$ phase, we can take the second factor in Eq. (7) to be a constant $=A$. Then we get

$$
T(t)=A \times \sin ^{2}[2 \Phi(t)+2 \alpha] .
$$

If we insert the expression for $\Phi$ from Eq. (6) into Eq. (8) we get

$$
T(t)=A \times \sin ^{2}\left[2 \alpha+4 \quad \arctan \left\{e^{t / \tau} \times \tan \left(\Phi_{0}\right)\right\}\right] .
$$

The most commonly used polarized- analyzer settings are $\alpha=0$ or $\alpha=\pi / 4$. We performed experiments with $\alpha=\pi / 4$. For this case, Eq. (9) will give a $T(t)$ curve as in Fig. 5, where we used the parameter value $\Phi_{0}=2 \mathrm{deg}$ for the initial azimuthal angle, and time is expressed as the dimensionless parameter $t / \tau$. As can be seen in the figure, the transmission curve gives two symmetric minima, corresponding to c director orientations $\Phi=\pi / 4$ and $\Phi=3 \pi / 4$. The delay time from the time of field reversal until the transmission changes appreciably is sensitive to the value of $\Phi_{0}$, while $\tau$ determines the width of the two dips in the intensity curve.

When comparing the curve in Fig. 5 with our experimentally recorded transmission curves (Fig. 3), we noted that the experimental curves do not show a distinct maximum between the two minima, but rather show one broad minimum, or a minor maximum between the minima. In order to explain this effect, we suggest that the main reason for the observed behavior is the nonuniformity of the electric field in the active electrode gap. Since the electrodes are very thin ITO coatings (about $1000 \AA$ ), the field lines will in principle be in the $z y$ plane, if we neglect edge effects due to the finite length of the electrode gap. The field will be stronger near 


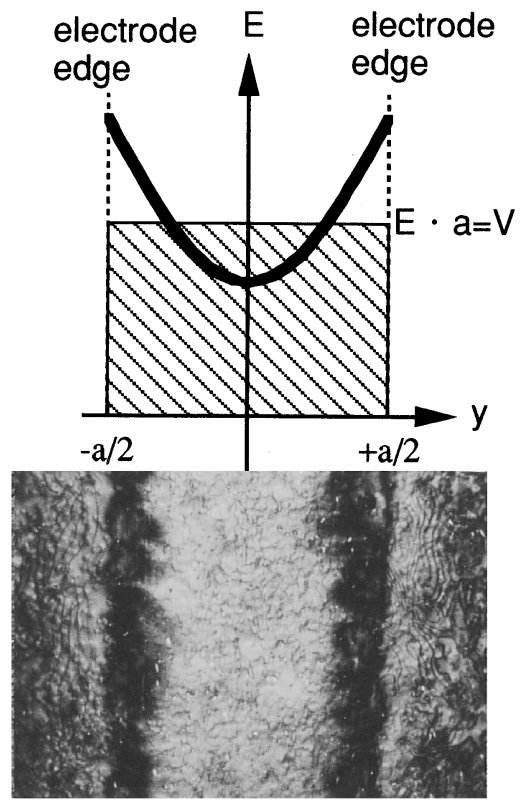

FIG. 6. Nonuniform electric field across the electrode gap.

the electrode edges, and weaker in the center of the active area. Such a field variation along $y$ is in agreement with our earlier observations ${ }^{4}$ of the switching time as a function of position along $y$ (measured in small spots). The switching time $\tau$ was found to have a maximum at the center of the electrode gap, corresponding to a minimum in the field $E$ according to the relation $\tau=\gamma_{\Phi} / P E$.

\section{B. Nonuniform electric field}

Since the thickness of the polymer films usually are in the $1-10 \mu \mathrm{m}$ range, while the distance between the electrodes is much larger, 500-1000 $\mu \mathrm{m}$, we simplify the analysis to include only the variation of the electric field along $y$, and neglect the small $z$ component. The electric field variation is illustrated in Fig. 6. The function $E(y)$ can be chosen in different ways to account for the field variation, but the form

$$
E(y)=E_{m} \times[1-\varepsilon \times \cos (\pi y / a)]
$$

or its approximation to second order,

$$
E(y)=E_{m} \times\left[1-\varepsilon\left\{1-(2 y / a)^{2}\right\}\right],
$$

will suitably describe the field variation along $y$, with $E_{m}$ being the field at the electrode edges and $\varepsilon$ a parameter describing the degree of field inhomogeneity. Referring to Fig. 6, the electric field $E$ should be integrated from $y=-a / 2$ to $y=a / 2$ to give the applied voltage:

$$
\int_{-a / 2}^{a / 2} E(y) d y=V .
$$

In the case of constant field, this just gives $a \times E=V$. If we choose the nonuniform field $E(y)$ according to Eq. (10), and perform the integration given by Eq. (12), we obtain

$$
E(y)=\frac{V}{a} \frac{1}{1-2 \varepsilon / \pi}[1-\varepsilon \times \cos (\pi y / a)],
$$
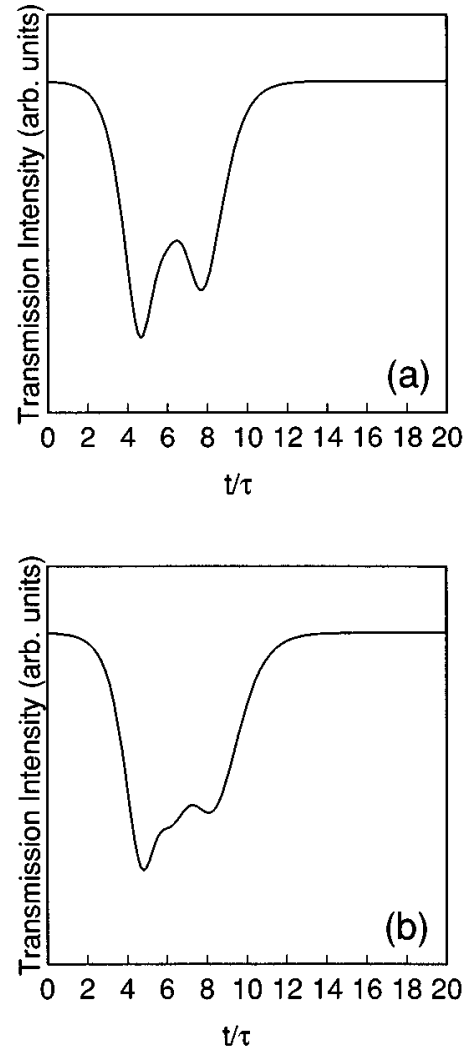

FIG. 7. Results from a simulation of the transmission during switching with square-wave voltage, taking into account the nonuniform electric field distribution through Eq. (16): (a) $T(t)=\int_{-0.15}^{-0.05} T(y, t) d y \quad(\varepsilon=0.55, y=$ $-0.15 \sim-0.05 \mathrm{~mm})$; (b) $T(t)=\int_{0.55}^{0.65} \int_{-0.15}^{-0.05} T(\varepsilon, y, t) d y d \varepsilon \quad(\varepsilon=0.55$ $\sim 0.65, y=-0.15 \sim-0.05 \mathrm{~mm})$.

which for small $\varepsilon$ can be written

$$
E(y)=(V / a)(1+2 \varepsilon / \pi)[1-\varepsilon \times \cos (\pi y / a)] .
$$

The spatial variation of $E$ leads to a spatial variation of the transmission during the switching process $T=T(y, t)$. If we measure the transmission in the active area in a small spot between $y=y_{1}$ and $y=y_{2}$, we must at every time instant integrate $T(y, t)$ between $y_{1}$ and $y_{2}$ to get the time dependence of the total transmission $T(t)$ through the spot:

$$
T(t)=\int_{y_{1}}^{y_{2}} T(y, t) d y
$$

with $T(y, t)$ given by

$$
\begin{aligned}
T(y, t)= & A \times \sin ^{2}\left[2 \alpha+4 \quad \arctan \left\{e^{t \times\left(P / \gamma_{\Phi}\right) \times E(y)}\right.\right. \\
& \left.\left.\times \tan \left(\Phi_{0}\right)\right\}\right] .
\end{aligned}
$$

Such integrations are shown in Fig. 7 for different values of the parameter $\varepsilon$ describing the field nonuniformity. It is seen that the distribution of switching times introduced by the model of Eq. (16) will drastically change the shape of the transmission curve. The model implies that the switching starts at the electrode edges and progresses towards the center, which is in accordance with observation of the switching in stroboscopic light. 
In the experimental results shown in Fig. 3, the more resolved peaks for the thinner cell can be understood if one assumes that the field uniformity is better for a thinner film, and thus that the parameter $\varepsilon$ is smaller. This gives a more narrow distribution of switching times across the measuring spot, so that the transmission curve will be more like the ideal one for a totally uniform switching. Our model can thus readily account for the shape of the switching peak itself. However, the very slow process observed when the azimuthal angle $\Phi$ approaches $\pi-\Phi_{0}$ is not reproduced, since Eq. (16) will produce symmetric forms of the $T(t)$ curve for times corresponding to $0<\Phi<\pi / 4$ and $3 \pi / 4<\Phi<\pi$. The asymmetric shape suggests a different dissipative mechanism at the final stage of switching compared to the initial state. Accounting for this type of dissipative asymmetry calls for an additional term in the equation of motion for the director [Eq. (4)]. Another reason for the asymmetry might be that the variation of the initial angle $\Phi_{0}$ with $y$, coming from the dependence of $E$ on $y$, is not included in the model in its present form, therefore the inhomogeneity of the rotation sense in the electrode gap due to the variation of the $\Phi_{0}$ is probable. On the other hand, the model, which essentially gives the transmission curve as an average of a distribution of different switching states, does correctly describe the essential features of the switching in terms of only one external parameter accounting for the field nonuniformity.

\section{MULTIELECTRODED SUBSTRATES}

Substrates with a pattern of interdigitated electrodes were used for spin-coating polymer films of $2 \mu \mathrm{m}$ thickness. Homeotropic films of excellent orientation were obtained in the same way as for the one-electrode gap substrates. The distance between the electrodes was $12 \mu \mathrm{m}$, the electrodes were $13 \mu \mathrm{m}$ wide, and the whole addressed area was $2 \times 2$ mm (cf. Fig. 2).

By applying a dc voltage of $5 \mathrm{~V}$, it was possible to induce a grating structure in the film, with an angle $\pi$ between the $\mathbf{c}$ directors in two adjacent addressed stripes. Between crossed polarizers, such an arrangement gives the same transmission for the adjacent stripes, which is seen in Fig. 8(a) as white bands with a thin black line between them, this line being a disclination wall. An interesting feature is that also over the electroded areas, where the field is zero, an alignment occurs for the $\mathbf{c}$ director, given by the alignment in the adjacent addressed area. This effect probably arises due to the weak orientational elasticity along the smectic layers, so that the orienting effect of the field stretches far outside the field area.

In order to optically distinguish between two adjacent stripes, one needs to use oblique incident light. One way to do this is to apply directional filtering in the microscope. This means that we choose to use only a part of the light cone supplied by the condenser to illuminate the sample. If the part of the light cone chosen approximately corresponds to one of the two director orientations in the stripes, then for these directions of the light rays there will be extinction between crossed polarizers, since these directions correspond to light propagation along the optic axis. For the other director orientation (with $\mathbf{c}$ director rotated an angle $\pi$ ), there will be
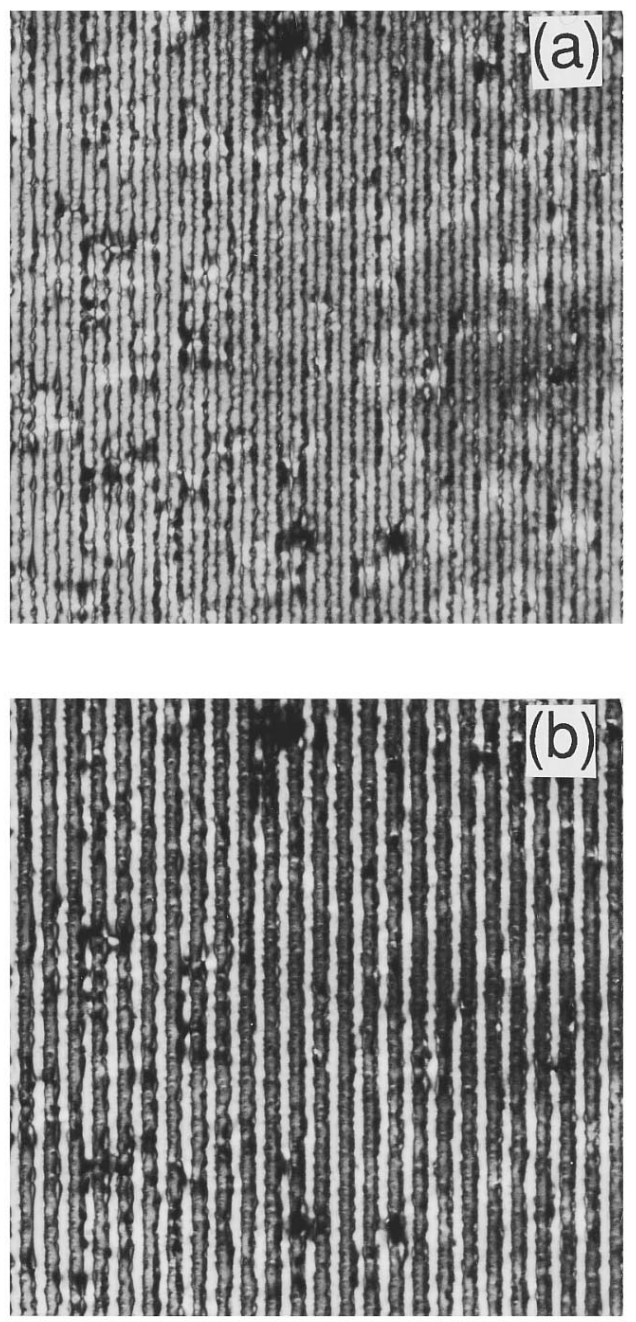

FIG. 8. (a) Microphotograph of $2 \mu \mathrm{m}$ film on the multielectroded substrate. (b) Same as in (a), but applying directional filtering.

transmission, since there will be an effective birefringence as seen by the oblique incident light. Such an incident-light condition was applied for the recording of Fig. 8(b).

The use of multielectroded substrates provides interesting possibilities for amplifying optical and electrical signals from the switching process. An example is show in Fig. 9,

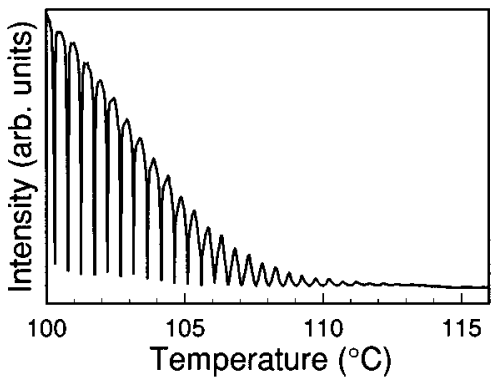

FIG. 9. Optical transmission recorded in the multielectroded $2 \mu \mathrm{m}$ film between crossed polarizers as a function of temperature. Triangular driving voltage of amplitude $40 \mathrm{~V}$ and frequency $0.017 \mathrm{~Hz}$. The onset of electroclinic switching at about $115^{\circ} \mathrm{C}$ is clearly seen, and the smectic $A-C^{*}$ transition at about $105^{\circ} \mathrm{C}$ is also shown. 
where the multielectroded cell was used to record the smectic $A-$ smectic $C^{*}$ phase transition by recording the transmitted intensity through crossed polarizers when applying a triangular voltage of amplitude $40 \mathrm{~V}$ and frequency 0.017 $\mathrm{Hz}$. The transition from electroclinic to ferroelectric switching is observed to occur at the temperature $T=105^{\circ} \mathrm{C}$, which agrees well with our results from differential scanning calorimetry (DSC) and electro-optical measurements in bulk-cell preparations. ${ }^{5}$ At about $113^{\circ} \mathrm{C}$ the onset of electroclinic switching is observable. This switching is characterized by an induced tilt angle being proportional to the applied electric field. For small tilt angle, which is the case here, this results in an optical transmission between crossed polarizers that is also linear in the applied field. Such linear switching is seen between about 113 and $105^{\circ} \mathrm{C}$. Below $105^{\circ} \mathrm{C}$ there is a temperature range, going down to about $102{ }^{\circ} \mathrm{C}$, where there is a mixed switching behavior, characterized of both electroclinic and cone switching. In this interval, the soft mode in the smectic $C^{*}$ phase influences the switching characteristics, so that the switching consists of two processes: at first a fast cone reorientation, and then a linear increase in tilt angle. Below about $102{ }^{\circ} \mathrm{C}$, the normal ferroelectric response is seen, with a pure cone reorientation and no soft-mode behavior.

\section{CONCLUSIONS}

In conclusion, we have demonstrated the electro-optic switching in spin-coated free-surface films of ferroelectric polymer liquid crystals. The switching model which we propose in this article takes into account the nonuniformity of the electric field in the electrode gap. The result is that the switching process in a finite measuring spot is given as an average of a distribution of different switching states. The theoretical model is able to describe the essential features of our experimental observations of the optical transmission during switching. Multielectroded films were also prepared, and although the films were used in this study mainly for detection of the smectic $A-C^{*}$ transition and the onset of electroclinic switching, the overall application potential of this type of electro-optic polymer films is obviously very large. As one example, a study of the use of multielectroded mesomorphic polymer films for electrically switchable phase gratings and color switches is currently underway. ${ }^{8}$

\section{ACKNOWLEDGMENTS}

This work was partly supported by the Swedish Natural Science Research Council. We gratefully acknowledge valuable comments by Professor M. Yamashita, Tokushima College of Technology.

${ }^{1}$ A. T. Royappa and M. F. Rubner; Langmuir 8, 3168 (1992).

${ }^{2}$ M. Onoda and K. Yoshino, Jpn. J. Appl. Phys. 34, L266 (1995).

${ }^{3}$ K. Skarp, K. Myojin, H. Moritake, M. Ozaki, K. Yoshino, and B. Helgee, J. Soc. Electron Mater. Eng. 3, 69 (1994).

${ }^{4}$ K. Skarp, S. Uto, K. Myojin, H. Moritake, M. Ozaki, K. Yoshino, and B. Helgee, Jpn. J. Appl. Phys. 34, 5433 (1995).

${ }^{5}$ B. Helgee, T. Hjertberg, K. Skarp, G. Andersson, and F. Gouda, Liq. Cryst. 18, 871 (1995)

${ }^{6}$ M. Ozaki, A. Tagawa, Y. Sadohara, S. Oda, and K. Yoshino, Jpn. J. Appl. Phys. 30, 2366 (1991).

${ }^{7}$ K. Skarp and M. Handschy, Mol. Cryst. Liq. Cryst. 165, 439 (1988).

${ }^{8}$ S. Uto, H. Moritake, M. Ozaki, K. Yoshino, K. Skarp, and B. Helgee (to be published). 